\title{
Initiation of aneurysms as a mechanical bifurcation phenomenon
}

\author{
Y.B. Fu ${ }^{1}$, G.A. Rogerson ${ }^{1}$, and Y.T. Zhang ${ }^{2}$ \\ ${ }^{1}$ Department of Mathematics, Keele University, ST5 5BG, U.K. \\ ${ }^{2}$ Department of Mechanics, Tianjin University, Tianjin 300072, China
}

May 23, 2011

\section{Abstract}

Recent studies on localized bulging in inflated membrane tubes have shown that the initiation pressure for the onset of localization is determined through a bifurcation condition. This kind of localization has also been shown to be much more sensitive to geometrical and material imperfections than classical sub-critical bifurcation into periodic patterns. We use these results to show that the initial formation of aneurysms in human arteries may also be modeled as a bifurcation phenomenon. This bifurcation interpretation could provide a theoretical framework under which different mechanisms leading to, or reducing the risk of, aneurysm formation can be assessed in a systematic manner. In particular, this could potentially help in assessing the integrity of aneurysm repairs.

Keywords: Aneurysms; membrane tubes; localization; bifurcation; stability.

\section{Introduction}

An aneurysm is a localized, blood-filled balloon-like bulge of a blood vessel. As the size of an aneurysm increases, there is an increasing risk of rupture, resulting in severe hemorrhage, other complications or even death. Aneurysm formation has broadly been associated with hereditary predisposition, old age and hypertension, and more specifically with defects in extracellular matrix maturation, increased degradation of elastin and collagen, aberrant cholesterol homeostasis, or enhanced production of angiotension peptides. However, how such factors manifest themselves in changing the mechanical behaviour of arteries before aneurysm formation is still not fully understood. Existing studies in the bio-mechanical community have largely focussed on modeling the material properties of mature aneurysm tissues and the growth of aneurysms when they have already formed; 
see, e.g., Humphrey and Rajagopal [1], Venkatasubramaniam et al. [2], Watton et al. [3], Baek et al. [4, 5], Vande Geest et al. [6], Vorp [7], Watton and Hill [8], and the references therein. Such studies are obviously important in guiding a clinician when to intervene when an aneurysm has been diagnosed. Our current study, however, is focussed on understanding the process leading to the initial formation of an aneurysm.

The geometrical similarity between a localized bulge in an inflated hyperelastic membrane tube and an arterial aneurysm is obvious, but the former problem is much better understood thanks to a large number of experimental, numerical and analytical studies, and the absence of uncertainty in the material modelling. When a hyperelastic membrane tube is inflated by pumping in air or water, a localized bulge will form when the internal pressure reaches a certain critical value. Once a bulge has been initiated, its early stage of growth is highly unstable, which takes place at decreasing pressure (and associated reduction of radius away from the center of the bulge). For almost all rubber-like materials, growth will stop when the bulge reaches a so-called Maxwell state which is stable. Further inflation will force the bulge to spread in both directions and will take place at a constant Maxwell pressure and constant maximum radius. The earliest documented observation of localized bulging in inflated membrane tubes seems to have been by Mallock [9]. For a selection of experimental, numerical and analytical studies, we refer to Yin [10], Chater and Hutchinson [11], Kyriakides and Chang [12, 13], and Shi and Moita [14]. Although this problem has been loosely referred to as a stability/bifurcation problem, its precise stability/bifurcation nature was not fully understood until very recently; see Fu et al. [15]. In this paper, the localized bulging was recognized as a nonlinear bifurcation problem (the corresponding linear bifurcation analysis incorrectly predicts the bifurcation mode as a uniform radial expansion), and it was shown that the initiation pressure may or may not equal the limiting pressure associated with uniform inflation depending on end conditions. Equality holds if, for instance, the ends are closed and any external axial force is fixed, but in the case of open ends localized bulging would occur before the limiting pressure is reached. Characterization of the entire inflation process and its stability was carried out in two subsequent papers $[16,17]$. In a more recent paper [18] it was further shown that the initiation of localized bulging in inflated membrane tubes is more sensitive to material and geometrical imperfections than classical subcritical bifurcations into sinusoidal patterns. The latter obeys Koiter's two-thirds power rule [19, 20] whereas the former obeys a square root rule. For instance, a localized wall thinning that corresponds to a $10 \%$ maximum wall thickness reduction can easily induce a $19 \%$ reduction in the critical circumferential stretch. Since for arteries the pressure is an exponential function of circumferential stretch, such a $19 \%$ reduction in stretch can reduce the critical pressure by many orders of magnitude. Such severe imperfection insensitivity has the potential to bring the critical pressure down to physiologically possible values and is crucial to our argument in the present paper. 
It is well known that arteries are inelastic: they show hysteresis when subjected to cyclic loading and unloading, and also exhibit typical viscoelastic behaviour (i.e. stress relaxation at a constant strain and strain creep at a constant stress), but for many practical purposes, including ours in the current paper, it suffices to model them as nonlinearly elastic and anisotropic. There is, however, a major difference between the behaviour of rubber-like materials and that of arteries, namely that for the former the strain energy function can be accurately described by an algebraic function (see, e.g., Ogden [21]), whereas for the latter the behaviour is typically exponential (see, e.g., Fung et al. [22]). Furthermore, arteries have a layered structure and behave anisotropically, and as a result there is much uncertainty in their mathematical modelling. Because of these differences, when aneurysm formation is interpreted as a bifurcation phenomenon it is not immediately clear whether the bifurcation condition has any solutions at all. Then to demonstrate that aneurysm formation can indeed be modeled as a bifurcation phenomenon, we need to first choose an appropriate material model, and then to verify that bifurcation can not only take place but also can be achieved at physiologically possible pressure values. A number of material models for arteries have been proposed in recent years; we refer to Humphrey [23] and Holzapfel and Ogden [24] for comprehensive reviews. In this paper, we select two representative material models. The first is the multi-layer structural model of Holzapfel et al. [25] which also gives material parameter data for carotid arteries of a young rabbit. The second is the single-layer arterial model proposed by Choi and Vito [26] with data provided by Vande Geest et al. [6] for a group of healthy but elderly human aortic arteries. It is known that aneurysms are rare in animals and more so among young animals, and that aneurysms can be induced by pathological changes in elderly humans. Our challenge is to show that the bifurcation interpretation can indeed capture, at least qualitatively, these basic facts.

The rest of this paper is organized as follows. In the next section, we summarize Holzapfel et al.'s [25] multi-layer structural model and show that for axi-symmetric deformations, provided each layer in an artery is hyperelastic, the composite artery is necessarily hyperelastic and we give the effective strain-energy function. This result paves the way for the application of $\mathrm{Fu}$ et al.'s [15] bifurcation condition which we briefly derive in Section 3 for completeness. We show that the bifurcation condition reduces to a determination of the zeros of the expression (3.11) by virtue of a local analysis of the ordinary differential equation (3.10). This bifurcation condition is then applied in Section 4 to the two material models mentioned in the previous paragraph. The paper is concluded with a summary and some additional remarks. 


\section{Governing equations}

Healthy arteries are composed of three clearly defined layers: the intima (the innermost layer), the media (the middle layer) and the adventitia (the outer layer). Each layer of the arterial wall may be considered as a composite reinforced by two families of fibres arranged in symmetrical helices [27]. With incompressibility assumed, the strain energy function $\Psi$ for each layer is a function of the seven invariants $I_{1}, I_{2}, I_{4}, I_{5}, I_{6}, I_{7}, I_{8}$ defined by (Spencer [28])

$$
\begin{gathered}
I_{1}=\operatorname{tr} C, \quad I_{2}=\frac{1}{2}\left(I_{1}^{2}-\operatorname{tr} C^{2}\right), \quad I_{4}=\boldsymbol{M} \cdot C \boldsymbol{M}, \quad I_{5}=\boldsymbol{M} \cdot C^{2} \boldsymbol{M}, \\
I_{6}=\boldsymbol{M}^{\prime} \cdot C \boldsymbol{M}^{\prime}, \quad I_{7}=\boldsymbol{M}^{\prime} \cdot C^{2} \boldsymbol{M}^{\prime}, \quad I_{8}=\boldsymbol{M} \cdot C \boldsymbol{M}^{\prime},
\end{gathered}
$$

where $C$ is the right Cauchy-Green strain tensor, and $\boldsymbol{M}$ and $\boldsymbol{M}^{\prime}$ are the directions of the two families of fibres in the reference configuration. The Cauchy stress tensor is then given by

$$
\begin{gathered}
\sigma=-p I+2 \Psi_{1} B+2 \Psi_{2}\left(I_{1} B-B^{2}\right)+2 \Psi_{4} \boldsymbol{m} \otimes \boldsymbol{m} \\
+2 \Psi_{5}(\boldsymbol{m} \otimes B \boldsymbol{m}+B \boldsymbol{m} \otimes \boldsymbol{m})+2 \Psi_{6} \boldsymbol{m}^{\prime} \otimes \boldsymbol{m}^{\prime} \\
+2 \Psi_{7}\left(\boldsymbol{m}^{\prime} \otimes B \boldsymbol{m}^{\prime}+B \boldsymbol{m}^{\prime} \otimes \boldsymbol{m}^{\prime}\right)+\Psi_{8}\left(\boldsymbol{m} \otimes \boldsymbol{m}^{\prime}+\boldsymbol{m}^{\prime} \otimes \boldsymbol{m}\right),
\end{gathered}
$$

where $p$ is the pressure associated with the constraint of incompressibility, $B$ is the left Cauchy-Green strain tensor, $\Psi_{i}=\partial \Psi / \partial I_{i}(i=1,2, \ldots, 8)$, and $\boldsymbol{m}=F \boldsymbol{M}, \boldsymbol{m}^{\prime}=F \boldsymbol{M}^{\prime}$ with $F$ being the deformation gradient.

We shall choose a common cylindrical polar coordinate system, with basis vectors $\boldsymbol{e}_{r}, \boldsymbol{e}_{\theta}, \boldsymbol{e}_{z}$, to describe vectors and tensors in both the current and reference configurations. Thus, we may write

$$
\boldsymbol{M}=\cos \phi \boldsymbol{e}_{\theta}+\sin \phi \boldsymbol{e}_{z}, \quad \boldsymbol{M}^{\prime}=\cos \phi \boldsymbol{e}_{\theta}-\sin \phi \boldsymbol{e}_{z}
$$

where $\phi$ is the constant angle between the collagen fibres and the circumferential direction.

We consider the problem of axi-symmetric inflation of a straight artery that has constant wall thickness $H$ and uniform mid-plane radius $R$ before inflation. Thus, in general, the axisymmetric deformed configuration may be described by

$$
r=r(Z), \quad z=z(Z)
$$

where $Z$ and $z$ are the axial coordinates of a representative material particle before and after inflation, respectively, and $r$ is the mid-plane radius after inflation.

Since the deformation is axially symmetric, the principal directions of stretch coincide with the lines of latitude, the meridian and the normal to the deformed surface. Denoting the unit vectors in these principal directions by $\boldsymbol{e}_{1}, \boldsymbol{e}_{2}, \boldsymbol{e}_{3}$, respectively, we have

$$
\boldsymbol{e}_{1}=\boldsymbol{e}_{\theta}, \quad \boldsymbol{e}_{2}=\cos \gamma \boldsymbol{e}_{z}+\sin \gamma \boldsymbol{e}_{r}, \quad \boldsymbol{e}_{3}=-\sin \gamma \boldsymbol{e}_{z}+\cos \gamma \boldsymbol{e}_{r}
$$




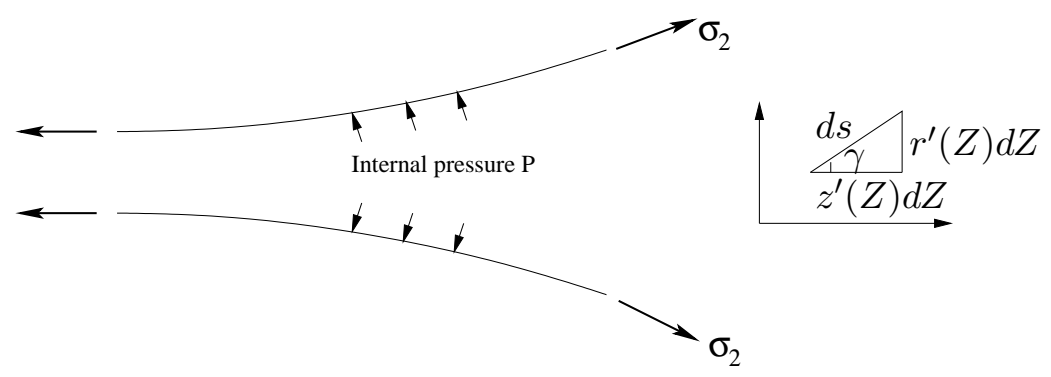

Figure 1: Axisymmetric deformation of an artery.

where $\gamma$ is the angle between the meridian and the $z$-direction; see Fig.1. The associated principal stretches are given by

$$
\lambda_{1}=\frac{r}{R}, \quad \lambda_{2}=\sqrt{r^{\prime 2}+z^{\prime 2}}, \quad \lambda_{3}=\frac{h}{H},
$$

where $h$ denotes the deformed wall thickness and the primes indicate differentiation with respect to $Z$. In the following analysis, we use $R$ as the unit of length, which is equivalent to setting $R=1$.

The deformation gradient $F$ may be written as

$$
F=\lambda_{1} \boldsymbol{e}_{1} \otimes \boldsymbol{e}_{\theta}+\lambda_{2} \boldsymbol{e}_{2} \otimes \boldsymbol{e}_{z}+\lambda_{3} \boldsymbol{e}_{3} \otimes \boldsymbol{e}_{r}
$$

See, e.g., Haughton [29]. It then follows that

$$
\boldsymbol{m}=\lambda_{1} \cos \phi \boldsymbol{e}_{1}+\lambda_{2} \sin \phi \boldsymbol{e}_{2}, \quad \boldsymbol{m}^{\prime}=\lambda_{1} \cos \phi \boldsymbol{e}_{1}-\lambda_{2} \sin \phi \boldsymbol{e}_{2},
$$

and so

$$
\begin{gathered}
I_{4}=\boldsymbol{m} \cdot \boldsymbol{m}=\lambda_{1}^{2} \cos ^{2} \phi+\lambda_{2}^{2} \sin ^{2} \phi=I_{6}, \\
I_{5}=\boldsymbol{m} \cdot B \boldsymbol{m}=\lambda_{1}^{4} \cos ^{4} \phi+\lambda_{2}^{4} \sin ^{4} \phi=I_{7} .
\end{gathered}
$$

We make the further assumption that the two families of fibres are mechanically equivalent and so the strain energy must be symmetric with respect to interchange of $I_{4}$ and $I_{6}$. This then implies that $\Psi_{4}=\Psi_{6}$ and $\Psi_{5}=\Psi_{7}$. With the use of the additional results

$$
\begin{gathered}
\boldsymbol{m} \otimes \boldsymbol{m}+\boldsymbol{m}^{\prime} \otimes \boldsymbol{m}^{\prime}=2 \lambda_{1}^{2} \cos ^{2} \phi \boldsymbol{e}_{1} \otimes \boldsymbol{e}_{1}+2 \lambda_{2}^{2} \sin ^{2} \phi \boldsymbol{e}_{2} \otimes \boldsymbol{e}_{2} \\
\boldsymbol{m} \otimes B \boldsymbol{m}+B \boldsymbol{m} \otimes \boldsymbol{m}+\boldsymbol{m}^{\prime} \otimes B \boldsymbol{m}^{\prime}+B \boldsymbol{m}^{\prime} \otimes \boldsymbol{m}^{\prime}=4 \lambda_{1}^{4} \cos ^{2} \phi \boldsymbol{e}_{1} \otimes \boldsymbol{e}_{1}+4 \lambda_{2}^{4} \sin ^{2} \phi \boldsymbol{e}_{2} \otimes \boldsymbol{e}_{2} \\
\boldsymbol{m} \otimes \boldsymbol{m}^{\prime}+\boldsymbol{m}^{\prime} \otimes \boldsymbol{m}=2 \lambda_{1}^{2} \cos ^{2} \phi \boldsymbol{e}_{1} \otimes \boldsymbol{e}_{1}-2 \lambda_{2}^{2} \sin ^{2} \phi \boldsymbol{e}_{2} \otimes \boldsymbol{e}_{2}
\end{gathered}
$$

we can see from (2.2) that $\sigma$ is co-axial with $B$ and that the three principal stresses are given by

$$
\sigma_{1}=2 \Psi_{1} \lambda_{1}^{2}+2 \Psi_{2}\left(\lambda_{1}^{2} \lambda_{2}^{2}+\lambda_{1}^{2} \lambda_{3}^{2}\right)+\left(4 \Psi_{4}+2 \Psi_{8}\right) \lambda_{1}^{2} \cos ^{2} \phi+8 \Psi_{5} \lambda_{1}^{4} \cos ^{2} \phi-p
$$




$$
\begin{gathered}
\sigma_{2}=2 \Psi_{1} \lambda_{2}^{2}+2 \Psi_{2}\left(\lambda_{1}^{2} \lambda_{2}^{2}+\lambda_{3}^{2} \lambda_{2}^{2}\right)+\left(4 \Psi_{4}-2 \Psi_{8}\right) \lambda_{2}^{2} \sin ^{2} \phi+8 \Psi_{5} \lambda_{2}^{4} \sin ^{2} \phi-p, \\
\sigma_{3}=2 \Psi_{1} \lambda_{3}^{2}+2 \Psi_{2}\left(\lambda_{1}^{2} \lambda_{3}^{2}+\lambda_{2}^{2} \lambda_{3}^{2}\right)-p .
\end{gathered}
$$

Similar expressions have previously been given by Ogden [30] for the case when the two families of fibres are symmetrically disposed in a plane.

With the use of the membrane assumption $\sigma_{3}=0$, we may eliminate $p$ from the above expressions and then show that

$$
\sigma_{1}=\lambda_{1} \frac{\partial \hat{\Psi}}{\partial \lambda_{1}}, \quad \sigma_{2}=\lambda_{2} \frac{\partial \hat{\Psi}}{\partial \lambda_{2}}
$$

where

$$
\hat{\Psi}\left(\lambda_{1}, \lambda_{2}\right)=\Psi\left(I_{1}, I_{2}, I_{4}, I_{5}, I_{6}, I_{7}, I_{8}\right),
$$

and $\lambda_{3}$ has been eliminated using $\lambda_{1} \lambda_{2} \lambda_{3}=1$.

For a 3-layered arterial model, simple consideration of equilibrium shows that the principal stresses in the composite artery are given by

$$
\sigma_{\alpha}=\sum_{i} \frac{H^{(i)}}{H} \sigma_{\alpha}^{(i)}=\lambda_{\alpha} \frac{\partial}{\partial \lambda_{\alpha}} \sum_{i} \frac{H^{(i)}}{H} \hat{\Psi}^{(i)}, \quad \alpha=1,2 ; \text { no summation on } \alpha,
$$

where the summation is over the three composite layers with superscript $(i)$ signifying association with the individual layers (for instance $H^{(i)}$ is the uniform thickness of the $i$-th layer). The above expression motivates the introduction of the following effective/homogenized strain-energy function:

$$
W\left(\lambda_{1}, \lambda_{2}\right)=\sum_{i} \frac{H^{(i)}}{H} \hat{\Psi}^{(i)}
$$

and in terms of it the expressions for the principal stresses have the same form as for the case of an isotropic and homogeneous hyperelastic membrane tube.

\section{Bifurcation condition}

The equilibrium equations can be obtained by minimizing the energy functional

$$
E=\int_{-L}^{L} W\left(\lambda_{1}, \lambda_{2}\right) 2 \pi R H d Z-P \int_{-L}^{L} \pi r^{2} z^{\prime} d Z
$$

where $L$ is the length of the artery in the un-inflated configuration and $P$ is the internal pressure; see Fig.1. On setting the first variation of $E$ to zero, we obtain

$$
\begin{gathered}
\left(\frac{H W_{2} r^{\prime}}{\lambda_{2}}\right)^{\prime}+\operatorname{Pr} z^{\prime}-H W_{1}=0, \\
\left(\frac{H W_{2} z^{\prime}}{\lambda_{2}}\right)^{\prime}-P r r^{\prime}=0
\end{gathered}
$$


where $W_{1}=\partial W / \partial \lambda_{1}, W_{2}=\partial W / \partial \lambda_{2}$. The equilibrium equation (3.3) can be integrated straight away to yield

$$
\frac{H W_{2} z^{\prime}}{\lambda_{2}}-\frac{1}{2} \operatorname{Pr}^{2}=\text { constant } \equiv C_{2},
$$

which reflects the fact that the resultant in the $Z$-direction at any cross section must be a constant. The two equilibrium equations also have another integral

$$
W-\lambda_{2} W_{2}=\text { constant } \equiv C_{1}
$$

as first noted by Pipkin [31].

Since we are only concerned with localized deformations, the artery can effectively be assumed to be infinite. On taking the limit $Z \rightarrow \infty$ in (3.2), (3.4) and (3.5), we obtain

$$
P=\frac{H W_{1}^{(\infty)}}{r_{\infty} z_{\infty}}, \quad C_{2}=H W_{2}^{(\infty)}-\frac{1}{2} P r_{\infty}^{2}, \quad C_{1}=W^{(\infty)}-z_{\infty} W_{2}^{(\infty)}
$$

where the superscript $(\infty)$ signifies evaluation at

$$
\lambda_{1}=r_{\infty}, \quad \lambda_{2}=z_{\infty}
$$

$r_{\infty}$ and $z_{\infty}$ being the radius and $z^{\prime}$ at infinity, respectively. In our subsequent analysis, $z_{\infty}$ is assumed to take a fixed value and $r_{\infty}$, related to pressure through $(3.6)_{1}$, is taken to be the single control parameter in our bifurcation analysis.

We note that the uniform solution (3.7) is always a solution. To investigate whether a non-uniform solution can bifurcate from this uniform solution or not, we write $r=r_{\infty}+y$ and assume that $|y| \ll 1$. Equation (3.5) can then be used to find the Taylor expansion

$$
\lambda_{2}=z_{\infty}+d_{1} y+\frac{1}{2} d_{2} y^{2}+\cdots
$$

and (3.4) used to find the Taylor expansion

$$
z^{\prime}=z_{\infty}+g_{1} y+\frac{1}{2} g_{2} y^{2}+\cdots
$$

where $d_{1}, d_{2}, g_{1}, g_{2}$ are constants that can easily be determined with the use of a symbolic manipulation package such as Mathematica [32]. On substituting these expansions into $\lambda_{2}=\sqrt{r^{\prime 2}+z^{\prime 2}}$, we obtain

$$
y^{\prime 2}=\omega\left(r_{\infty}, z_{\infty}\right) y^{2}+O\left(y^{3}\right)
$$

where

$$
\omega\left(r_{\infty}, z_{\infty}\right)=\frac{\left(r_{\infty} W_{11}^{(\infty)}-W_{1}^{(\infty)}\right) W_{22}^{(\infty)} z_{\infty}^{2}-r_{\infty}\left(W_{1}^{(\infty)}-W_{12}^{(\infty)} z_{\infty}\right)^{2}}{r_{\infty} z_{\infty} W_{12}^{(\infty)} W_{22}^{(\infty)}}
$$

and $W_{12}=\partial^{2} W / \partial \lambda_{1} \partial \lambda_{2}$ etc, with superscripts $(\infty)$ signifying evaluation at the state (3.7). The solution of $(3.10)$ changes character when $\omega\left(r_{\infty}, z_{\infty}\right)=0$. It can be shown that this is 
the condition for the onset of localizations [15]. Previously this bifurcation condition has been derived by Haughton and Ogden [33] as the condition for axi-symmetric buckling of a membrane tube at zero axial mode number (or equivalently infinite tube length). More recently, an alternative derivation of this bifurcation condition was given by Rodríguez and Merodio [34] who also explored certain aspects of the bifurcation interpretation of aneurysm formation.

\section{Numerical results}

\subsection{Results based on Holzapfel et al.'s [25] multi-layer model}

Following Holzapfel et al. [25], we ignore the effect of the intima and assume that the behaviour of each of the two other layers may be modeled by a strain energy function of the form

$$
\Psi=\mu\left(I_{1}-3\right)+\frac{k_{1}}{2 k_{2}} \sum_{\alpha=4,6}\left(\exp \left[k_{2}\left(I_{\alpha}-1\right)^{2}\right]-1\right),
$$

where $\mu$ and $k_{1}$ are material parameters with the dimension of stress, and $k_{2}$ is a dimensionless material parameter. It is understood that the material parameters $\mu, k_{1}, k_{2}, \phi$ take different values for the media and adventitia, respectively. Holzapfel et al. [25] gave 18 sets of values for these parameters by fitting this model to the experimental data and results given by Fung et al. [22] and Chuong and Fung [35] for the carotid arteries of a young rabbit. Similar values for human arteries do not seem to be available in the literature, although we note that parameter values for human coronary arteries have been given by Holzapfel et al. [36] for the extended strain energy function

$$
\Psi=\mu\left(I_{1}-3\right)+\frac{k_{1}}{2 k_{2}} \sum_{\alpha=4,6}\left(\exp \left\{k_{2}\left[(1-\rho)\left(I_{1}-3\right)^{2}+\rho\left(I_{\alpha}-1\right)^{2}\right]\right\}-1\right),
$$

where $\rho \in[0,1]$ is an additional parameter. This extended strain energy function will be discussed in the final section.

We use the command "ContourPlot" in Mathematica to plot $\omega\left(r_{\infty}, z_{\infty}\right)=0$ in the $\left(r_{\infty}, z_{\infty}\right)$-plane. We find that this bifurcation condition has a solution only for the 9 th set of material parameters, that is when

$$
\begin{gathered}
\mu^{(a)}=0, \quad k_{1}^{(a)}=6.1998 \mathrm{kPa}, \quad k_{2}^{(a)}=0.7520, \quad \phi^{(a)}=45^{\circ}, \\
\mu^{(m)}=0, \quad k_{1}^{(m)}=4.94 \mathrm{kPa}, \quad k_{2}^{(m)}=0.6246, \quad \phi^{(m)}=0^{o},
\end{gathered}
$$

where superscripts $(a)$ and $(m)$ correspond to the adventitia and media, respectively. The corresponding solution is shown in Fig.2. Interestingly, this is the only set in which the media fibres are in the circumferential direction. For the three choices $z_{\infty}=$ $1,1.3,1.6$, the corresponding bifurcation values of $r_{\infty}$ and the associated pressures are 


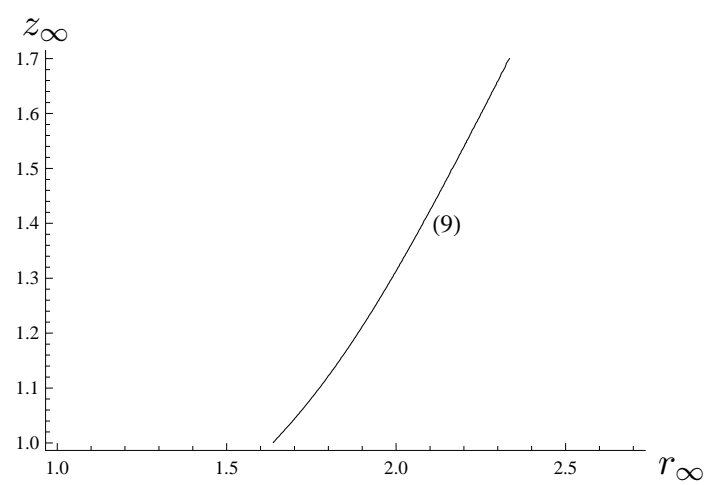

Figure 2: Solution of $\omega\left(r_{\infty}, z_{\infty}\right)=0$ when the artery is described by the two-layer model. The label (9) on the curve signifies association with the 9 th set of material parameters in Table 1 of [25].

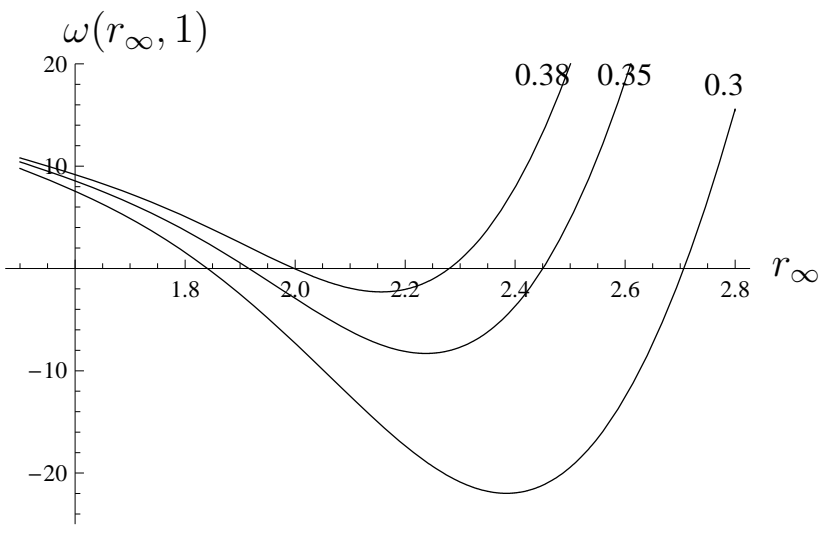

Figure 3: Variation of $\omega\left(r_{\infty}, 1\right)$ when the values of $k_{2}^{(1)}$ and $k_{2}^{(2)}$ are simultaneously multiplied by $0.38,0.35,0.3$, respectively.

$(1.636,434 \mathrm{mmHg}),\left(1.988,2.25 \times 10^{4} \mathrm{mmHg}\right),\left(2.251,3.36 \times 10^{6} \mathrm{mmHg}\right)$, respectively. To bring each of these three pressures down to $120 \mathrm{mmHg}$, the required reductions in $r_{\infty}$ are 11\%, 25\%,35\%, respectively. Fung et al. [22] gave 1.6 as the approximate axial in situ stretch for the carotid artery. If this is the case, then bifurcation is unlikely to occur unless pathological changes can also induce a reduction in the axial stretch.

Although bifurcation is not possible for the other 17 sets of material parameters, we now give an indication on how much the material parameters need to be varied in order to induce bifurcation. We first consider the effect of reducing the values of $k_{2}^{(a)}$ and $k_{2}^{(m)}$ by a common factor. For the first set of material parameters given in [25], it is found that $\omega\left(r_{\infty}, 1\right)$ begins to have a zero when the above material values are reduced by a factor of 0.3935 . In Fig.3 we have shown how the first zero of $\omega\left(r_{\infty}, 1\right)$ moves to the left as the material values are subject to more reductions. It is found that as we increase 


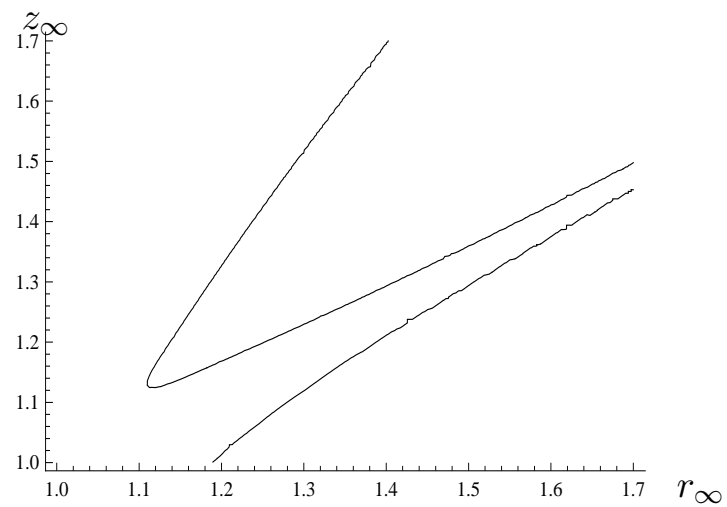

Figure 4: Solution of $\omega\left(r_{\infty}, z_{\infty}\right)=0$ for the Choi and Vito [26] material model

$z_{\infty}$ gradually, it becomes increasingly more and more difficult to induce a bifurcation. For example, when $z_{\infty}=1.4,1.6$, respectively, the required minimum reduction factor becomes 0.1 and 0.06 , respectively.

We have also considered the effect of reducing $k_{1}^{(a)}$ and $k_{1}^{(m)}$ by a common factor while keeping the other material parameters unchanged. It is found that this mechanism of inducing aneurysm formation is much less efficient. For example, when $z_{\infty}=1$, the required reduction factor is 0.0025545 with the associated zero occurring at $r_{\infty}=1.8$.

\subsection{Numerical results based on Choi and Vito's [26] single-layer model}

Vande Geest et al. [6] carried out a series of biaxial experiments on human abdominal aortic aneurysm tissues and compared their mechanical properties with those of agematched healthy abdominal aorta tissues. They fitted their experimental data to the following strain-energy function proposed by Choi and Vito's [26]:

$$
W=b_{0}\left\{\mathrm{e}^{\frac{1}{2} b_{1} E_{\theta \theta}^{2}}+\mathrm{e}^{\frac{1}{2} b_{2} E_{z z}^{2}}+\mathrm{e}^{b_{3} E_{\theta \theta} E_{z z}}-3\right\}
$$

where the $b$ 's are material constants and the $E$ 's are the components of the Green-Lagrange strain tensor $E=(C-I) / 2$ referred to cylindrical polar coordinates $(r, \theta, z)$ in the reference configuration. They gave 8 sets of parameter values for healthy abdominal aorta tissues. Again using the command "ContourPlot" in Mathematica to plot $\omega\left(r_{\infty}, z_{\infty}\right)=0$ in the $\left(r_{\infty}, z_{\infty}\right)$-plane, we find that $\omega\left(r_{\infty}, z_{\infty}\right)=0$ has solutions for all the 8 sets of parameter values. Fig.4 shows a typical plot of these solutions for their first set of parameter values given by

$$
b_{0}=0.13 \mathrm{kPa}, \quad b_{1}=391.6, \quad b_{2}=302.6, \quad b_{3}=309.8 .
$$

Plots of $\omega\left(r_{\infty}, z_{\infty}\right)=0$ for the other 7 sets of material parameters are qualitatively 
similar, and in particular they all have the double-branch behaviour in Fig.4. It is not clear from [6] what the axial pre-stretch is in the human arteries considered, and so we shall consider some typical values. For $z_{\infty}=1,1.3,1.6$, respectively, we find that the first bifurcation value of $r_{\infty}$ is equal to $1.19,1.19,1.35$, with corresponding pressure equal to $7.62 \times 10^{4} \mathrm{mmHg}, 4.61 \times 10^{10} \mathrm{mmHg}, 8.94 \times 10^{43} \mathrm{mmHg}$, respectively. These pressure values are clearly unrealistic. However, the large pressure values are due to the fact that the pressure is an exponentially growing function of $r_{\infty}$ for each fixed $z_{\infty}$, and to bring these pressures down to $120 \mathrm{mmHg}$, we only need to reduce the critical values of $r_{\infty}$ by $6.7 \%, 14 \%$, and $25 \%$, respectively. As shown by Fu and Xie [18], such reductions can be achieved by geometrical and/or material imperfections such as localized weakening of collagen fibres.

\section{Conclusion}

We have considered two material models to argue that the initial formation of aneurysms can be modeled as a bifurcation phenomenon. Using the single-layer model proposed by Choi and Vito [26], with material parameters given by Vande Geest et al. [6] for healthy human aortic arteries, we have demonstrated that bifurcation can be induced by geometrical and material imperfections. This is consistent with the established view that localized weakening of collagen fibres serves as a precursor to aneurysm formation. For the multi-layer model proposed by Holzapfel et al. [25], with material parameters given for carotid arteries of a young rabbit, our calculations show that bifurcation is much harder to achieve. This is consistent with the well-known result that aneurysms are very rare in animals [37].

We observe that Fu and Xie's [18] claim that "a 10\% maximum wall-thinning can induce a $19 \%$ reduction in the critical circumferential stretch" was based on a convenient choice of localized wall-thinning profile, and they did not make any attempt to find the types of imperfections that will induce maximum reduction in the critical circumferential stretch. Also, it is well-known that a localized bulge can be maintained at a much lower pressure than the initiation pressure. Thus, the amount of reductions in the initiation pressure quoted in our discussion in the previous section is not the maximum possible, and bifurcations are probably easier to induce than we have shown, especially if dynamic effects are also taken into account.

Finally, we wish to point out that the bifurcation condition is very sensitive to the choice of the material model. To illustrate this point, we have also solved the bifurcation condition for two additional material models. The first is the following strain energy function proposed by Chuong and Fung [35]:

$$
W=\frac{1}{2} c\left\{\mathrm{e}^{Q}-1\right\}
$$




$$
Q=b_{1} E_{\theta \theta}^{2}+b_{2} E_{z z}^{2}+b_{3} E_{r r}^{2}+2 b_{4} E_{\theta \theta} E_{z z}+2 b_{5} E_{z z} E_{r r}+2 b_{6} E_{r r} E_{\theta \theta},
$$

where $c$ and the $b$ 's are material constants. Chuong and Fung [35] obtained 18 sets of values for these constants by fitting their model to the experimental results of Fung et al [22] for the carotid arteries of five normal rabbits. In fact the 18 sets of material parameters given by Holzapfel et al. [25] are obtained by requiring their model and Chuong and Fung's [35] model to give the same response when each artery is subjected to bi-axial stretching. Holzapfel et al. [25] demonstrated that their model and Chuong and Fung's [35] model then predicted very similar behaviour for uniform inflation. However, we find that these two models give very different bifurcation results. Firstly, according to [35], the bifurcation condition does not have any solution for the 9th set of material parameters, but instead it has solutions for the 4th, 6th, 8th, 15th, 16th and 17th sets of material parameters; see Fig. 5. Secondly, the bifurcation prediction for the 4th, 6th, 8th, 15 th, and 17th sets of parameters does not seem to be realistic: the corresponding values of $r_{\infty}$, and hence the pressure, is too low, which makes aneurysm formation too easy. The prediction for the 16th set looks feasible: at $z_{\infty}=1.6$, the corresponding $r_{\infty}$ and pressure are 1.88 and $263 \mathrm{mmHg}$, respectively. We also observe that for the 8th, 16th and 17th sets of parameter values, the denominator in (3.11) has zeros for values of $r_{\infty}$ close to unity, signifying violation of the strong ellipticity condition. This undesirable feature of Chuong and Fung's [35] model has previously been pointed out by Wilber and Walton [38]. These additional calculations serve to highlight the fact that very accurate material modelling is crucial if the bifurcation condition is expected to give reliable predictions about aneurysm formation.
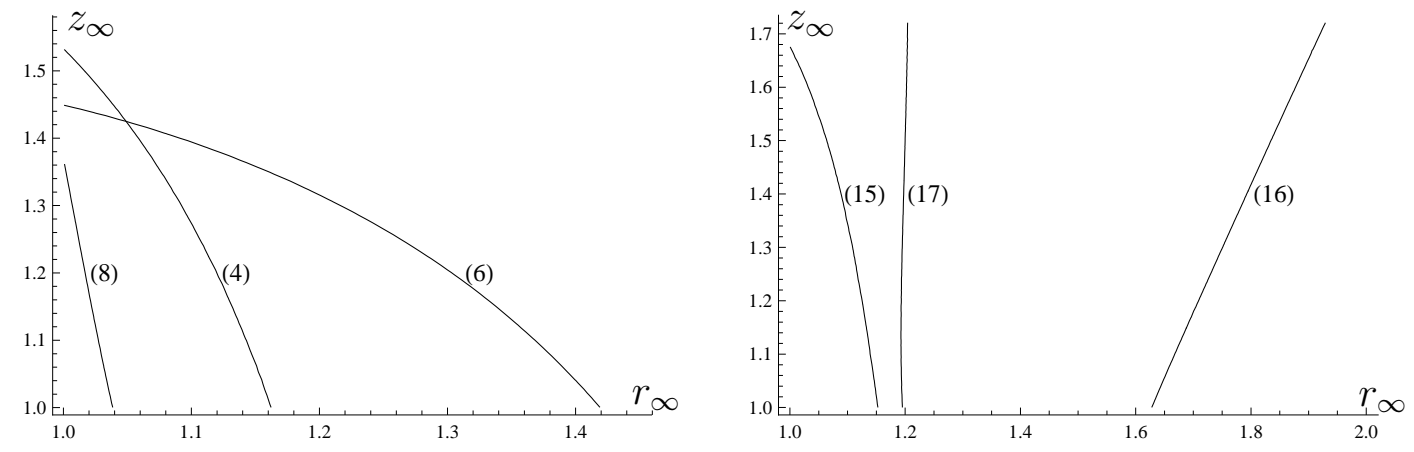

Figure 5: Solution of $\omega\left(r_{\infty}, z_{\infty}\right)=0$ when the artery is described by Chuong and Fung's [35] model. The label (4), for instance, indicates association with the 4th set of material material parameters given by Chuong and Fung's [35].

We finally consider the material model (4.2) for which Holzapfel et al.[36] have given 13 sets of parameter values for human coronary arteries with nonatherosclerotic intimal thickening. It is found that the bifurcation condition does not have a solution for any set of the parameter values. This result and those given in Section 4.2 are consistent with the 
fact that abdominal aortic aneurysms are more common than other forms of aneurysms.

\section{Acknowledgements}

This work was initially supported by an EPSRC "Bridging the Gaps" grant (EP/F033125/1) and later by an EPSRC grant (EP/G051704/1). We thank the referees for their constructive comments and suggestions.

\section{References}

1. J.D. Humphrey, K.R. Rajagopal, A constrained mixture model for growth and remodeling of soft tissues, Math. Model Meth. Appl. Sci. 12 (2002) 407-430.

2. A.K. Venkatasubramaniam, M.J. Fagan, T. Mehta, K.J. Mylankal, B. Ray, G. Kuhan, I.C. Chetter, P.T. McCollum, A comparative study of aortic wall stress using finite element analysis for ruptured and non-ruptured abdominal aortic aneurysms, Eur. J. Vasc. Endovasc. Surg. 28 (2004) 168-176.

3. P.N. Watton, N.A. Hill, M. Heil, A mathematical model for the growth of the abdominal aortic aneurysm. Biomechan Model Mechanobiol 3 (2004) 98-113.

4. S. Baek, K.R. Rajagopal, J.D. Humphrey, Competition between radial expansion and thickening in the enlargement of an intracranial saccular aneurysm, Journal of Elasticity 80 (2005) 13-31.

5. S. Baek, K.R. Rajagopal, J.D. Humphrey, A theoretical model of enlarging intracranial fusiform aneurysms. Transactions of the ASME, 128 (2006) 142-149.

6. J.P. Vande Geest, M.S. Sacks, D.A. Vorp, The effect of aneurysm on the biaxial mechanical behavior of human abdominal aorta, J. Biomech. 39 (2006) 1324-1334.

7. D.A. Vorp, Biomechanics of abdominal aortic aneurysm, J. Biomech. 40 (2007) 1887-1902.

8. P.N. Watton, N.A. Hill, Evolving mechanical properties of a model of abdominal aortic aneurysm, Biomechan Model Mechanobiol 8 (2009) 25-42.

9. A. Mallock, Note on the instability of India-rubber tubes and balloons when distended by fluid pressure, Proc. Roy. Soc. Lond. A 49 (1891) 458-463.

10. W.-L. Yin, Non-uniform inflation of a cylindrical elastic membrane and direct determination of the strain energy function, J. Elast. 7 (1977) 265-282. 
11. E. Chater, J.W. Hutchinson, On the propagation of bulges and buckles, ASME J. Appl. Mech. 51 (1984) 269-277.

12. S. Kyriakides, Y.-C. Chang, On the inflation of a long elastic tube in the presence of axial load, Int. J. Solids Struct. 26 (1990) 975-991.

13. S. Kyriakides, Y.-C. Chang, The initiation and propagation of a localized instability in an inflated elastic tube, Int. J. Solid Struct. 27 (1991) 1085-1111.

14. J. Shi, G.F. Moita, The post-critical analysis of axisymmetric hyper-elastic membranes by the finite element method, Comput. Methods Appl. Mech. Engrg. 135 (1996) 265-281.

15. Y.B. Fu, S.P. Pearce, K.K. Liu, Post-bifurcation analysis of a thin-walled hyperelastic tube under inflation, Int. J. Non-Linear Mech. 43 (2008) 697-706.

16. S.P. Pearce, Y.B. Fu, Characterisation and stability of localised bulging/necking in inflated membrane tubes, IMA J. Appl. Math. 75 (2010) 581-602.

17. Y.B. Fu, Y.X. Xie, Stability of localized bulging in inflated membrane tubes under volume control, Int. J. Eng. Sci. 48 (2010) 1242-1252.

18. Y.B. Fu, Y.X. Xie, Effects of imperfections on localized bulging in inflated membrane tubes, Phil. Trans. Roy. Lond., submitted.

19. W.T. Koiter, On the stability of elastic equilibrium, Thesis Delft (in Dutch, 1945), English translation: NASA TT F-10 833, 1967.

20. J.W. Hutchinson, W.T. Koiter, Postbuckling theory, Appl. Mech. Rev. 23 (1970) 1353-1365.

21. R.W. Ogden, Large deformation isotropic elasticity-on the correlation of theory and experiment for incompressible rubber-like solids, Proc. R. Soc. Lond. A 326 (1972) 565-584.

22. Y.C. Fung, K. Froneck, P. Patitucci, Pseudoelasticity of arteries and the choice of its mathematical expression, Am. J. Physiol. 237 (1979) H620-H631.

23. J.D. Humphrey, Mechanics of the arterial wall: review and directions, Crit. Rev. Biomed. Eng. 23 (1995) 1-162.

24. G.A. Holzapfel, R.W. Ogden, Constitutive modelling of arteries, Proc. R. Soc. A 466 (2010) 1551-1597. 
25. G.A. Holzapfel, T.C. Gasser, R.W. Ogden, Comparison of a multi-layer structural model for arterial walls with a Fung-type model, and issues of material stability, Trans. ASME 126 (2004) 264-275.

26. H.S. Choi, R.P. Vito, Two-dimensional stress-strain relationship for canine pericardium, J. Biomech. Eng. 112 (1990) 153-159.

27. G.A. Holzapfel, T.C. Gasser, R.W. Ogden, A new constitutive framework for arterial wall mechanics and a comparative study of material models, J. Elast. 60 (2000) 1-48.

28. A.J.M. Spencer, Constitutive theory for strongly anisotropic solids, in: A.J.M. Spencer (ed.), Continuum Theory of the Mechanics of Fibre-reinforced Composites, CISM Courses and Lectures No. 282, Springer-Verlag, Wien, 1984, pp 1-32.

29. D.M. Haughton, Elastic membranes, in: Y.B. Fu and R.W. Ogden (eds), Nonlinear Elasticity: Theory and Applications, Cambridge University Press, Cambridge, 2001, pp 233-267.

30. R.W. Ogden, Elements of the theory of finite elasticity, in: Y.B. Fu and R.W. Ogden (eds), Nonlinear Elasticity: Theory and Applications, Cambridge University Press, Cambridge, 2001, pp 1-57.

31. A.C. Pipkin, Integration of an equation in membranes theory, ZAMP 19 (1968) 818-819.

32. S. Wolfram, The Mathematica Book, Addison-Wesley, California, 2004.

33. D.M. Haughton and R.W. Ogden, Bifurcation of inflated circular cylinders of elastic material under axial loading. I. Membrane theory for thin-walled tubes, J. Mech. Phys. Solids 27 (1979), 179-212.

34. J. Rodríguez and J. Merodio, A new derivation of the bifurcation conditions of inflated cylindrical membranes of elastic material under axial loading. Application to aneurysm formatioin, Mech. Re. Commun. 38 (2011) 203-210.

35. C.J. Chuong, Y.C. Fung, Three-dimensional stress distribution in arteries, J. Biomech. Eng. 105 (1983) 268-274.

36. G.A. Holzapfel, G. Sommer, C.T. Gasser, P. Regitnig, Determination of layerspecific mechanical properties of human coronary arteries with nonatherosclerotic intimal thickning and related constitutive modeling, Am. J. Physiol. Heart Circ. Physiol. 289 (2005) H2048-H2058. 
37. Ch. Kim, H. Kikuchi, N. Hashimoto, F. Hazama, H. Kataoka, Establishment of the experimental conditions for inducing saccular cerebral aneurysms in primates with special reference to hypertension, Acta Neurochir. 96 (1989) 132-136.

38. J.P. Wilber, J.R. Walton, The convexity properties of a class of constitutive models for biological soft tissues, Math. Mech. Solids 7 (2002) 217-235. 